\title{
Predictive Value of Localized Stenosis of the Main Pancreatic Duct for Early Detection of Pancreatic Cancer
}

\author{
Yoshihide Kanno ${ }^{1}$, Shinsuke Koshita', Takahisa Ogawa ${ }^{1}$, Hiroaki Kusunose ${ }^{1}$, Kaori Masu', Toshitaka Sakai ${ }^{1}$ Keisuke Yonamine ${ }^{1}$, \\ Yujiro Kawakami', Yuki Fujii', Kazuaki Miyamoto', Toji Murabayashi', Fumisato Kozakai', Jun Horaguchi' ${ }^{1}$, Yutaka Noda', \\ Masaya Oikawa ${ }^{3}$, Takaho Okada $^{3}$ and Kei Ito ${ }^{1}$ \\ ${ }^{1}$ Department of Gastroenterology, Sendai City Medical Center, Sendai, ${ }^{2}$ Natori Chuo Clinic, Sendai, ${ }^{3}$ Department of Gastroenterological \\ Surgery, Sendai City Medical Center, Sendai, Japan
}

Background/Aims: In this study, we aimed to evaluate the predictive value of localized stenosis of the main pancreatic duct (MPD) for early detection of pancreatic cancer.

Methods: Among 689 patients who underwent endoscopic retrograde pancreatography from January 2008 to September 2018,19 patients with MPD findings were enrolled. These patients showed findings for indicating suspicious pancreatic cancer at an early stage (FiCE); FiCE was defined as a single, localized stenosis in the MPD without a detectable mass (using any other imaging methods) and without other pancreatic diseases, such as definite chronic pancreatitis, intraductal papillary mucinous neoplasm, and autoimmune pancreatitis. Final diagnoses were established by examining resected specimens or through follow-up examinations after an interval of $>5$ years.

Results: Among 19 patients with FiCE, 11 underwent surgical resection and 8 were evaluated after a $>5$-year observation period. The final diagnosis of the MPD stenosis was judged to be pancreatic cancer in 9 patients (47\%), including 3 with intraepithelial cancer, and to be a non-neoplastic change in 10 . The sensitivity, specificity, and accuracy of preoperative pancreatic juice cytology were $75 \%, 100 \%$, and $88 \%$, respectively.

Conclusions: The predictive value of FiCE for pancreatic cancer prevalence was $47 \%$. Histological confirmation with pancreatic juice cytology is necessary before surgical resection. Clin Endosc 2019;52:588-597

Key Words: Early pancreatic cancer; Endoscopic retrograde cholangiopancreatography; Intraductal papillary mucinous neoplasm; Intraepithelial cancer; Pancreatic juice cytology

\section{INTRODUCTION}

Although pancreatic cancer is a relatively infrequent malignancy, it is lethal and has a high mortality rate because curative treatment of pancreatic cancer is extremely difficult. ${ }^{1,2}$ The age-standardized incidence and mortality rates of pancreatic

Received: December 21, 2018 Revised: March 7, 2019

Accepted: March 7, 2019

Correspondence: Yoshihide Kanno

Department of Gastroenterology, Sendai City Medical Center, Tsurugaya 5-22-1, Miyagino-ku, Sendai, Miyagi 983-0824, Japan

Tel: +81-22-252-1111, Fax: +81-22-252-9431, E-mail: yoshi-hk@openhp.or.jp ORCID: https://orcid.org/0000-0002-2044-5483

cc This is an Open Access article distributed under the terms of the Creative Commons Attribution Non-Commercial License (http://creativecommons.org/ licenses/by-nc/3.0) which permits unrestricted non-commercial use, distribution, and reproduction in any medium, provided the original work is properly cited. cancer were 9.0 and 7.4 per 100,000 population, and it was the fourth leading cause of cancer-related death in 2013 in Japan. ${ }^{3}$ Similarly, the American Cancer Society estimated that 55,440 patients would be diagnosed with pancreatic cancer and that 44,330 deaths would be caused by pancreatic cancer in $2018{ }^{4}$ An analysis of the 30-year cancer registry in Japan found that the overall 5 -year survival rate was about $13 \%$ over the last 8 years. ${ }^{5}$ However, identification of pancreatic cancers at an early stage has been reported to result in better survival rates. ${ }^{5}$ According to the Japan Pancreatic Cancer Registry, the 5-year survival rates in patients with the Union for International Cancer Control Stage 0, Stage IA, and Stage IB pancreatic cancers were $85.8 \%, 68.7 \%$, and $59.7 \%$, respectively. Therefore, clinicians have been struggling to find early, small pancreatic cancers. 
Similar to other epithelial neoplasms, pancreatic cancer arises from the epithelium of pancreatic ducts. Morphological changes related to pancreatic cancer begin within the pancreatic duct rather than within the pancreatic parenchyma. Imaging findings indicative of pancreatic cancer at an early stage have recently been reported. ${ }^{2,6-8}$ According to several reports, pancreatic cancers have been detected at an early stage based on the presence of fine, short stenosis of the main pancreatic duct (MPD) ${ }^{2,9,10}$ However, cases with such MPD irregularities caused by spontaneous inflammation, i.e., non-neoplastic changes, are often encountered in daily practice. The prevalence of pancreatic cancer at the stenotic site is unknown. We retrospectively evaluated the predictive reliability of MPD findings that indicate pancreatic cancer at the early stage.

\section{MATERIALS AND METHODS}

\section{Patients}

We intentionally performed endoscopic retrograde pancreatography (ERP) in 689 patients from January 2008 to September 2018. From this population, we extracted data of patients who underwent ERP for examination of localized MPD stenosis that was detected during other examinations (Fig. 1, Table 1). To evaluate only slight changes in MPD that potentially indicated a tiny neoplasm, including an intraep- ithelial cancer, we excluded patients with a detectable mass (detected using imaging techniques other than ERP). Patients with abnormal MPD-related findings due to definite chronic pancreatitis, intraductal papillary mucinous neoplasms, and autoimmune pancreatitis were also excluded. In addition, patients with multiple stenoses were excluded because these findings indicate chronic inflammatory changes rather than

Table 1. Criteria for Defining Main Pancreatic Duct Findings for Indicating Suspicious Pancreatic Cancer at an Early Stage in This Study

Inclusion criteria
- Localized stenosis of the MPD
- Evaluation of the MPD by an intended ERP
Exclusion criteria
- A mass that is detectable on other imaging examinations
- Multiple stenosis of the MPD
- Accompaniment of the following diseases
Intraductal papillary mucinous neoplasms of the pancreas
Autoimmune pancreatitis or IgG4-related disease
Definite chronic pancreatitis, including pancreatic stones
History of physical injury to the pancreas
- State after surgical intervention related to the pancreas

ERP, endoscopic retrograde pancreatography; MPD, main pancreatic duct.

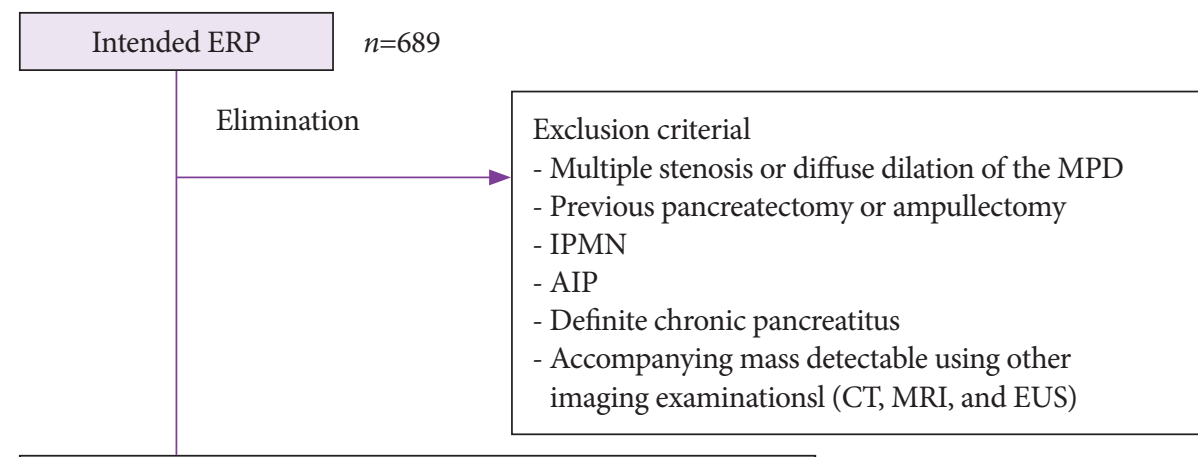

\begin{tabular}{|l|l|}
\hline \multicolumn{2}{|c|}{ Single stenosis on the MPD without a mass (defined as FiCE) } \\
\hline Elimination & $\begin{array}{l}\text { Not definitely diagnosed becouse; } \\
\text { (1) the lesion was not resected } \\
\text { (2) the lesion was not changed to obvious malignancy } \\
\text { (3) the lesion was not followed up for }>5 \mathrm{yr}\end{array}$ \\
\hline
\end{tabular}

Final diagnosis

Analyzed in this study $n=19$

Fig. 1. Study flow chart. AIP, autoimmune pancreatitis; CT, computed tomography; ERP, endoscopic retrograde pancreatography; EUS, endoscopic ultrasonography; FiCE, findings for indicating suspicious pancreatic cancer at an early stage; IPMN, intraductal papillary mucinous neoplasms; MPD, main pancreatic duct; MRI, magnetic resonance imaging. 
neoplasms.

The final diagnosis of the etiology of MPD stenosis was defined as being (1) malignant or benign on the basis of histological diagnosis in cases involving surgical resection, (2) malignant when an obviously malignant tumor appeared at the site during follow-up without resection, and (3) benign when the abnormality had improved or had not changed after the 5-year follow-up. When improvement or lack of change was difficult to determine, the patient was excluded. Patients who did not undergo endoscopic ultrasound (EUS), dynamic computed tomography (CT), magnetic resonance cholangiopancreatography, or ERP after the 5-year follow-up were not diagnosed with benign stenosis and were excluded from the study, even if clinical course, laboratory data, and transabdominal ultrasound (US) findings did not indicate an obvious malignancy in the pancreas.

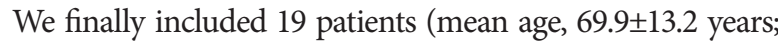
male, 10) who had a single, localized MPD stenosis without a detectable mass (detected using other imaging methods) in this study.

\section{Outcome measurements}

The main outcome was the predictive reliability of MPD findings for indicating suspicious pancreatic cancer at an early stage (FiCE). The criteria for a FiCE were (1) a single, localized stenosis of MPD without a mass on US, EUS, CT, or magnetic resonance imaging (MRI), and (2) the absence of clinical evidence for other MPD etiologies, such as intraductal papillary mucinous neoplasms, autoimmune pancreatitis, definite chronic pancreatitis, pancreatic stones, previous surgical intervention, and past physical injury (Table 1). Localized stenosis was defined as an obvious decrease in MPD diameter, compared with the diameters of both the ampullary and tail sides of the MPD. The predictive value was estimated using the percentage of patients with pancreatic neoplasms at the stenotic site among those with FiCE.

Secondary outcome measures were detailed findings related to the MPD, prevalence of tumor invasion on histological evaluation, stage of the malignancy, and diagnostic outcomes of cytology using pancreatic juice samples obtained during ERP examination.

The degree of MPD stenosis was estimated using ERP in which the contrast agent was injected from the ampullary side of the stenosis; the degree of MPD stenosis was classified into three groups: (1) complete obstruction (contrast agent could not be injected beyond the stenosis); (2) a severe thread-like stricture (contrast agent could advance beyond the stenosis); and (3) mild stenosis with a gentle change in MPD diameter. MPD diameter at the tail side of the stenosis was classified as follows: (1) "highly dilated", diameter $>2$ times the MPD diameter at the ampullary side; (2) "moderately dilated", diameter $<2$ times the MPD diameter at the ampullary side; and (3) "no dilation", diameter less than that of the MPD on the ampullary side.

Pancreatic juice was obtained using a cannula for a 0.035inch guidewire with a side hole (PR-104Q; Olympus Co., Tokyo, Japan) or a double lumen catheter for 0.035-inch and 0.025-inch guidewires (Uneven cannula; Piolax Medical Devices, Inc., Yokohama, Japan). Before aspiration of pancreatic juice, the stenotic site was rubbed with a guidewire to scratch off the epithelial cells. Lavage was performed with $40-60 \mathrm{~mL}$ saline while using a double lumen catheter. The obtained samples were prepared using the cell-block method and were evaluated with hematoxylin-eosin and periodic acid-Schiff/ alcian blue staining; if necessary, additional immunohistochemistry, such as staining for p53, Ki67, and MUC1, was also performed. Histological diagnosis was confirmed by an expert pathologist specializing in pancreatobiliary pathology (YN) and by another experienced pathologist who worked as a consultant at our center at that time.

Cytological diagnosis was classified as positive and negative. Cells judged as being definitely cancerous, those strongly suspected of being cancerous, and those that were difficult to judge but were consistent with cancer, i.e., results corresponding to Papanicolaou Class V, IV, and IIIb, were defined as being cytologically positive, whereas the others were defined as being cytologically negative.

The presence of a pancreatic mass was evaluated using EUS, enhanced dynamic CT, and MRI if the patient did not have a contraindication to the examination. EUS was performed using radial-arrayed (GF-UM2000, GF-UE260; Olympus) or convex-arrayed (GF-UCT260; Olympus) echoendoscopes by an expert endosonographer who had performed more than 1,000 examinations or by a trainee under an expert's supervision.

\section{Ethics}

Written informed consent was obtained for surgery and examinations, such as enhanced CT, EUS, and ERP. This retrospective study was approved by the Institutional Review Board of Sendai City Medical Center.

\section{RESULTS}

\section{Diagnostic yield of FiCE}

Among the 19 enrolled patients with FiCE, 11 underwent surgical resection (\#1-\#11 in Table 2) and the other 8 were followed up without surgery (\#12-\#19).

The final diagnosis of MPD stenosis was pancreatic cancer 


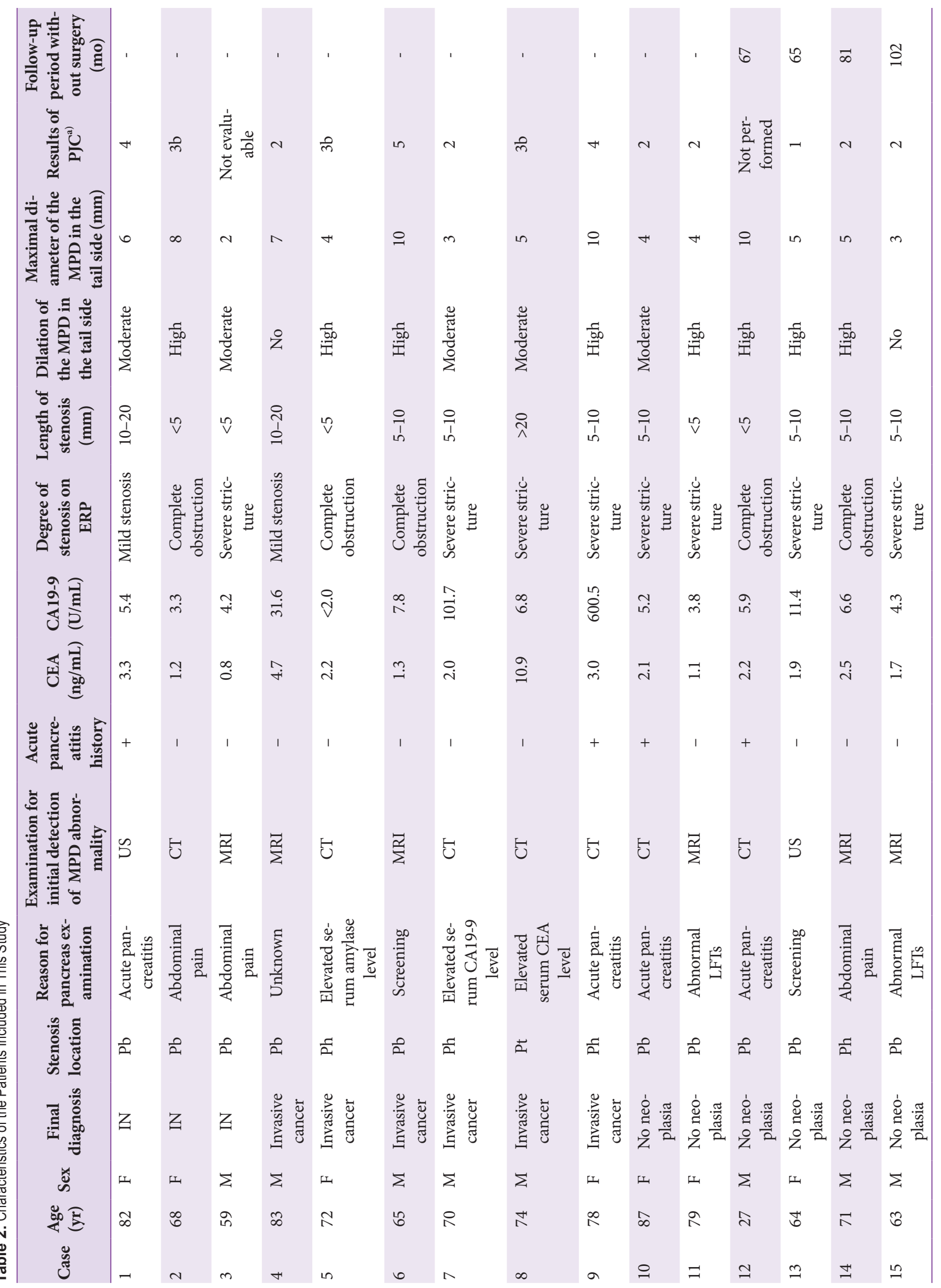




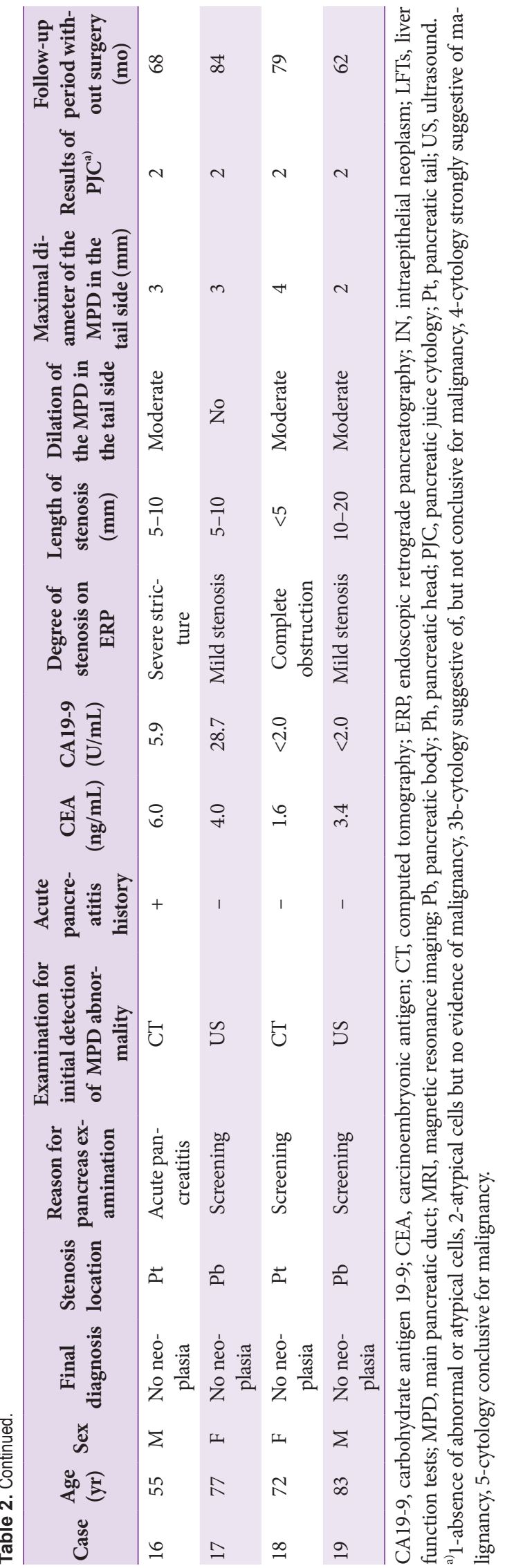

in 9 of the 11 patients who underwent surgery (Fig. 2) and a non-neoplastic change in the remaining 2 patients (Fig. 3A, B). Among the 9 patients who underwent surgery for malignant stenosis, 3 (\#1, \#4, and \#5) did not undergo resection immediately after ERP (Fig. 3C-I). Patient \#1 underwent resection for pancreatic cancer after a 14-month interval because she had previously undergone resection for simultaneously detected lung cancer. Patients \#4 and \#5 did not undergo immediate resection owing to negative cytology results; however, they underwent surgery 42 and 5 months later, respectively, because a mass detected at the stenosis site was diagnosed as malignant via EUS-guided fine needle aspiration cytology.

In the remaining 8 patients, MPD stenosis was judged as being non-neoplastic after clinical follow-up of $>5$ years (median follow-up period, 74 months; range, 62-102 months). No patients were found to have malignancies at the MPD stenosis site after clinical follow-up without resection.

Overall, MPD FiCE was finally diagnosed as a pancreatic cancer-related change in 9 patients (47\%) and a non-neoplastic change in 10 patients. In other words, the predictive value of FiCE for prevalence of pancreatic cancer was $47 \%$.

Of the 9 patients with pancreatic cancer, 3 were diagnosed with intraepithelial adenocarcinoma (high-grade pancreatic intraepithelial neoplasia [HG-PanIN]) and the remaining 6 with invasive ductal adenocarcinoma, based on examination of resected specimens. Mean size of the invasive region was $12.7 \pm 8.8 \mathrm{~mm}$ in the 6 patients with invasive cancer. The final cancer stages were as follows: 0, 3 patients; IA, 2 patients; IB, 2 patients; and IIB, 2 patients. Although no patients had distal metastases, Stage IIB cancers were accompanied by metastases to a pancreatic regional lymph node. Although EUS, dynamic $\mathrm{CT}$, and MRI were performed for all enrolled patients, no masses were identified, even during retrospective review. In the 2 patients who showed invasive regions sized $>20 \mathrm{~mm}$, the invasive regions were not reported to be round, dense, or well-demarcated, possibly due to the difficulty in detection during imaging examinations (Fig. 4).

\section{MPD findings}

MPD stenosis was located at the pancreatic head, body, and tail in 2, 6, and 1 patients with cancer and in 1, 7, and 2 patients without cancer, respectively (no statistical difference) (Table 2). Moreover, degree of stenosis and tail-side dilation did not differ between patients with and without cancer. Lengths of the stenosis were also similar in both groups.

\section{Other clinical findings}

Abdominal examinations, through which MPD findings were detected, were performed due to acute pancreatitis in 5 patients and abdominal pain in 3 patients. The remaining 11 
patients, including 4 in whom abnormalities were detected via screening US, did not have any symptoms.

Serum carcinoembryonic antigen (CEA) levels were within the normal limit $(<5.0 \mathrm{ng} / \mathrm{mL})$ in all patients except two whose CEA levels were slightly elevated due to smoking. Elevated serum carbohydrate antigen 19-9 (CA19-9) levels (normal limit, $<37.0 \mathrm{U} / \mathrm{mL}$ ) were observed in two patients with invasive cancer (\#7 and \#9), but serum CA19-9 levels decreased to normal after resection.

No patients exhibited any clinical features indicative of pancreatic cancer, including anorexia, weight loss, new-onset diabetes, indigestion, and pyrexia.

\section{Accuracy of pancreatic juice cytology}

The sensitivity, specificity, and accuracy of pancreatic cytology performed on samples from 18 patients were $67 \%$, $100 \%$, and $83 \%$, respectively. The sample obtained from a patient with a HG-PanIN (\#3 in Table 2) was not sufficient for histological evaluation. Excluding this sample, the sensitivity, specificity, and accuracy of cytology were $75 \%, 100 \%$, and $88 \%$, respectively. Two patients received false-negative diagnoses (\#4 and \#7); in these patients, the investigated cells were judged as only having mild atypia that could not be defined as malignant change. Five patients, who did not have neoplastic cells in their pancreatic juice samples (\#3,\#4,\#7, \#10, and \#11)
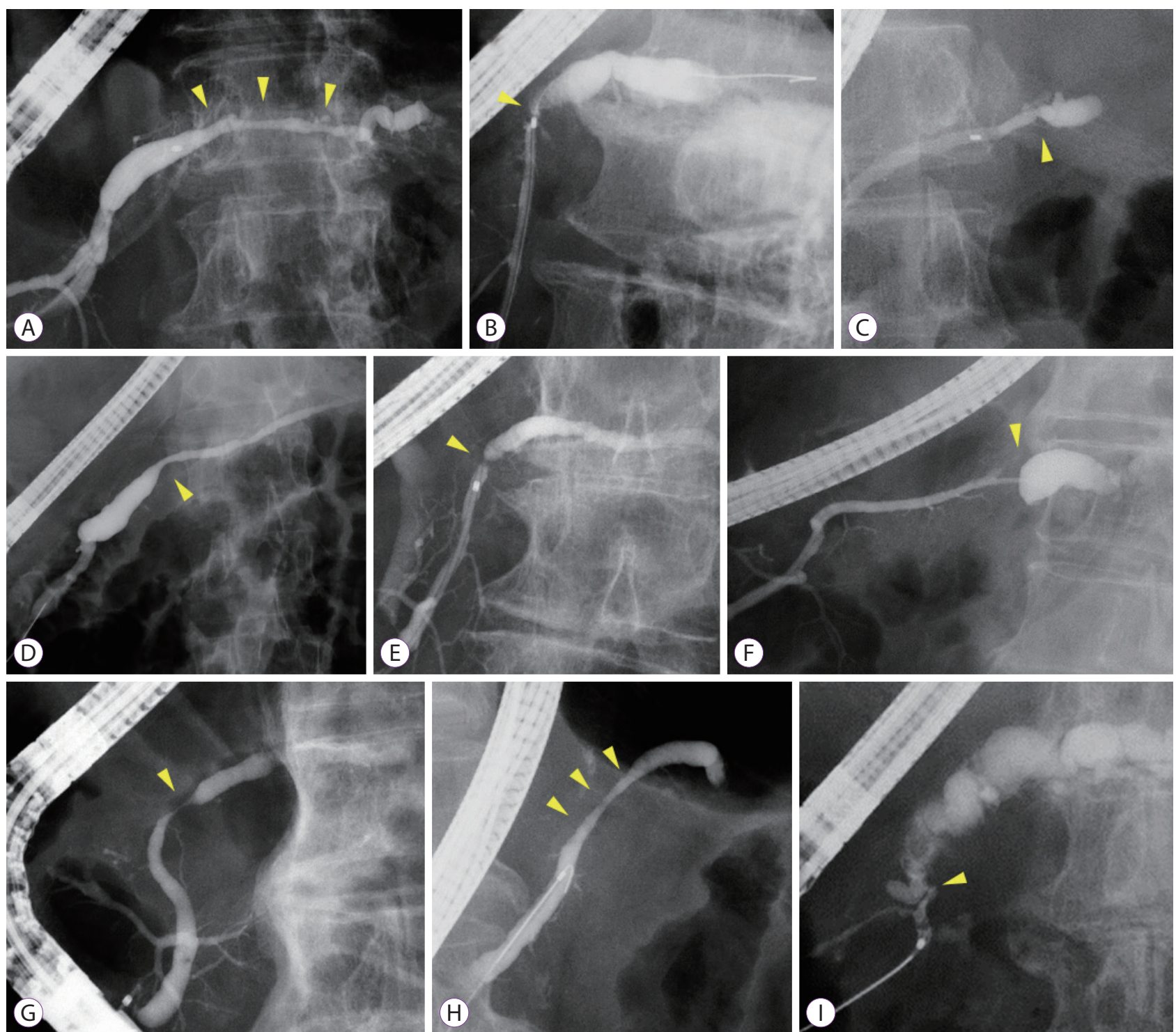

Fig. 2. Endoscopic retrograde pancreatography in patients diagnosed with malignant main pancreatic duct (MPD) stenosis with (findings for indicating suspicious pancreatic cancer at an early stage) without masses that are detectable via other imaging examinations. (A-I) show cases \#1-\#9 (Table 2) in a sequential order. (B, E, F) (Cases \#2, \#5, and \#6, respectively) were obtained after a catheter was advanced into the tail side of the MPD along a guidewire, although contrast was not injected into the tail side as the catheter could not be advanced beyond the stenosis. Arrowheads indicate stenosis of the MPD. 
desired and underwent surgical resection after giving full informed consent. Pancreatic juice cytology was not performed for one patient (\#12) because the procedure was ceased before obtaining sufficient pancreatic juice due to patient's non-cooperation.

Post-procedural pancreatitis with mild severity, according to Consensus Criteria, ${ }^{11,12}$ was observed in 3 patients (16\%).

\section{Prognosis}

All 10 patients who underwent surgery for neoplastic stenosis were still alive during the median follow-up period of 9 months (range, 3-68 months), which lasted till the last checkup before this analysis. However, one Stage-IA cancer patient who showed an invasive region of $8 \mathrm{~mm}$ developed metastatic recurrence in the lung, although he has been alive for 18 months after surgery without chemotherapy (\#4 in Tables 2, 3). Another patient showed metachronous pancreatic cancer in the remnant pancreatic head (\#6). This patient underwent surgical resection of the remnant pancreas for the Stage-IA cancer 39 months after the first surgery and has be alive for 17 months post-second surgery without recurrence.

The 2 patients who underwent distal pancreatectomy despite a non-neoplastic stenotic change are alive without surgery-related sequelae 280 (\#10) and 75 (\#11) days, respectively, post-surgery. All 8 patients who did not undergo surgery are alive after a median follow-up period of 74 months.

\section{DISCUSSION}

Although identification of small cancers is generally associated with a better prognosis, in pancreatic cancer, the 5-year survival rate has been reported to be only around $80 \%$, even when the tumor size is $<10 \mathrm{~mm}$. In other words, $20 \%$ of patients die even if the mass is sized $<10 \mathrm{~mm}$. Therefore, pancreatic cancer with parenchymal invasion is related to mortality,
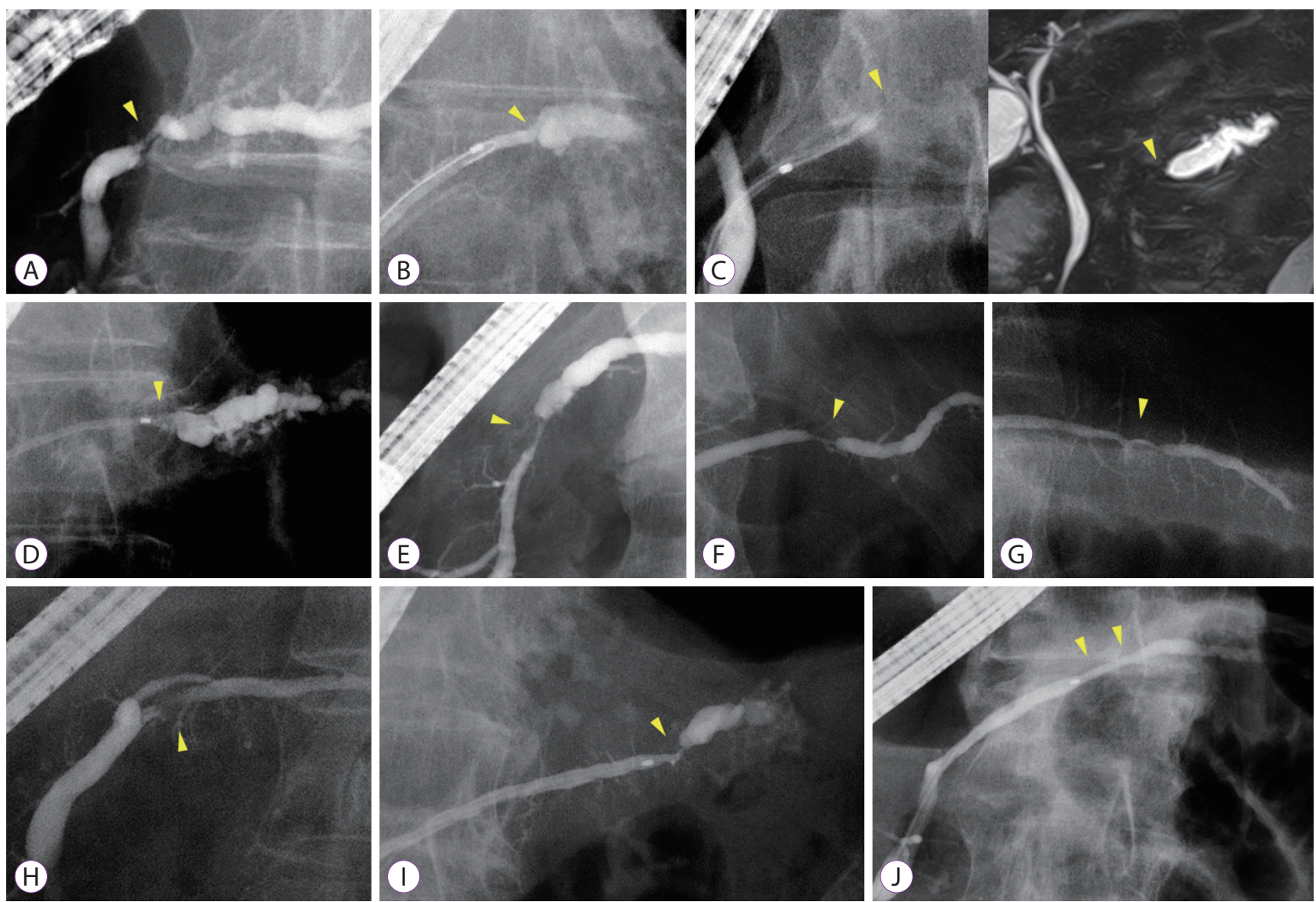

Fig. 3. Endoscopic retrograde pancreatography and magnetic resonance cholangiopancreatography (MRCP) in patients diagnosed with benign main pancreatic duct (MPD) stenosis with (findings for indicating suspicious pancreatic cancer at an early stage) without masses that are detectable via other imaging examinations. (AI) show Cases \#10-\#18 (Table 2) in a sequential order. Because neither contrast nor a catheter could be advanced to the tail side of the MPD in Case \#12, MRCP is additionally shown to show tail-side pancreatography (C). (B, D, J) (Cases \#11, \#13, and \#17, respectively) were obtained after a catheter was advanced into the tail side of the MPD along a guidewire, although contrast was not injected into the tail side before the catheter was advanced beyond the stenosis. 
even when the mass is small. This is the reason why, at present, early stage pancreatic cancer is not defined by any classification or guidelines. For ensuring the consistency of definition of early pancreatic cancer with other gastrointestinal cancers, in which early stage is defined as cancer without invasion into the muscular layer, we think that early pancreatic cancer can be defined as cancer without invasion, i.e., intraepithelial cancer.

Intraepithelial cancer in the pancreatic duct may induce intraluminal changes, such as stenosis at the lesion and dilation of the upstream duct. When a lesion develops in the MPD, it can be identified by detecting changes in the MPD via imaging examinations, even if the lesion itself cannot be visualized. Recently, detection of intraepithelial pancreatic cancer by investigation of abnormal MPD findings has been reported. ${ }^{2,9,10,13,14}$ At present, such MPD findings are the sole indicator for intraepithelial pancreatic cancer.

However, non-cancer cases with such MPD findings are often encountered. The predictive accuracy or rate of negative diagnosis of such findings has not been determined. We attempted to estimate the predictive value of these MPD findings in this study. Although this study is limited by the small sample size and retrospective setting, we observed an intermediate rate of negative diagnosis.

Confirming benignity is extremely difficult when the patient is followed-up without surgical resection. In this study, the minimal time for diagnosing benignity was defined to be 5 years in which the clinical course remained consistent and MPD findings did not change on imaging examination. Although this definition is not perfect, the period could not be extended to avoid inclusion of patients who developed new tumors at the stenotic site. Benign MPD stenosis is caused by inflammation and can induce chronic inflammation; therefore, new malignancies can occur at that site. Because we cannot conduct a "perfect" study in which all patients undergo resection owing to ethical reasons, the definition used in this
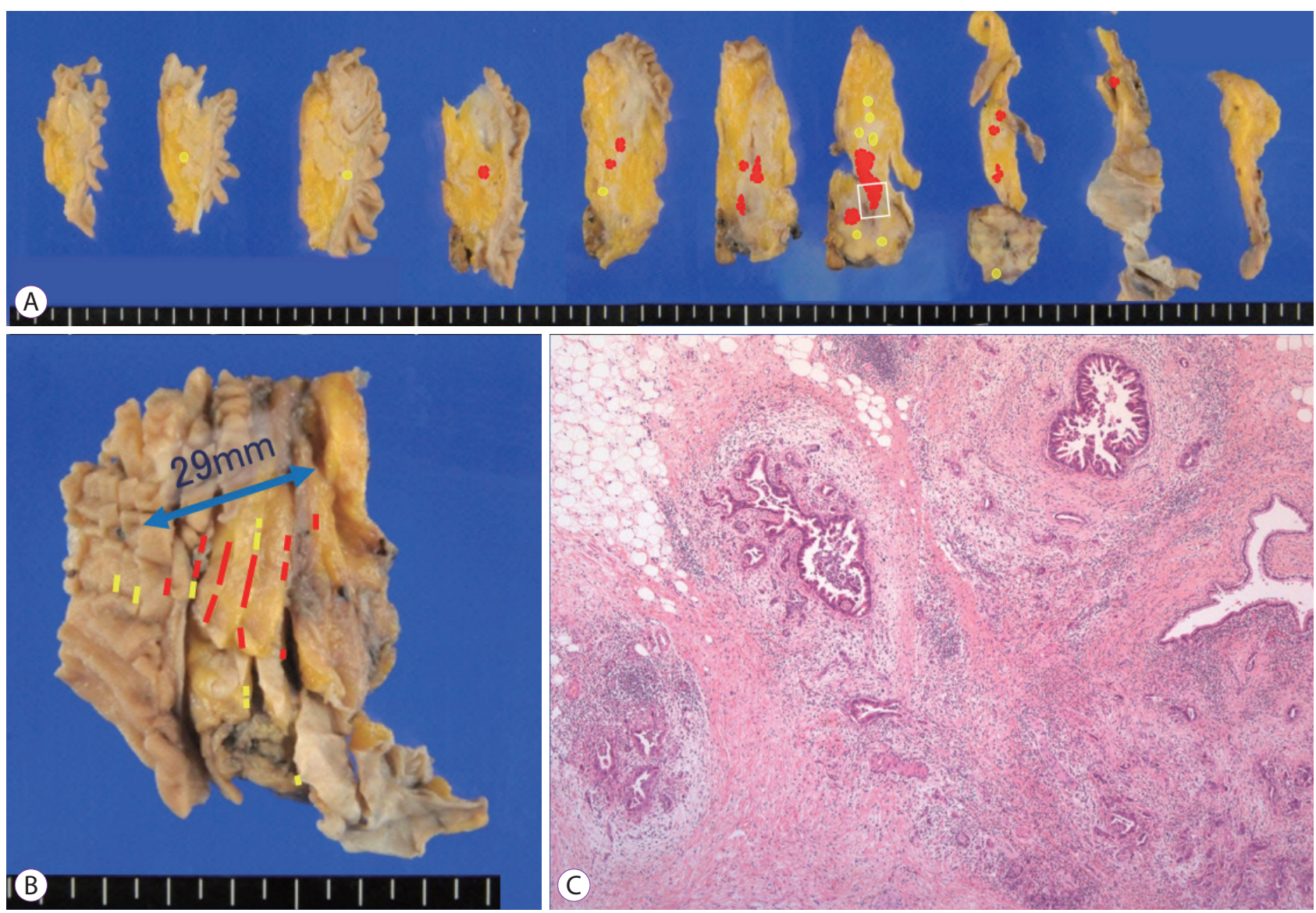

Fig. 4. Cancer invasion, with the invasive region being $27 \mathrm{~mm}$ in size (Case \#9 in Table 2). (A) Mapping through divided faces; (B) Mapping through cutting lines; (C) Microscopic examination of hematoxylin-eosin-stained specimen $(\times 10)$ showing a whitish, hard region, indicating that invasive cancer was not identified macroscopically on the divided faces. Microscopic evaluation shows that the invasive region (red color) was not round, dense, or well-demarcated, possibly due to the difficulty in detection during imaging examinations. The invasive region is indicated in red and the high-grade pancreatic intraepithelial neoplasia is indicated in yellow. The white box in $(A)$ indicates the location of $(C)$. The blue arrow indicates the main pancreatic duct near the stenosis. 


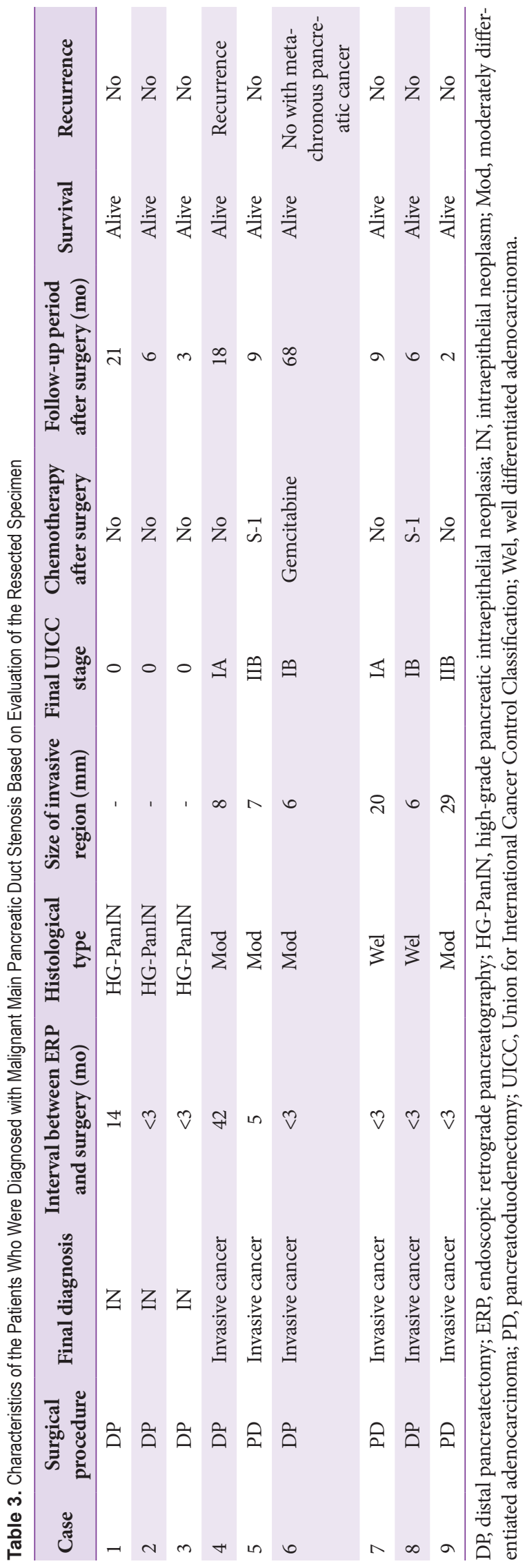

study is appropriate.

However, this limitation might provide misleading values in terms of cancer prevalence because we excluded patients MPD FiCE who were followed up without surgery for $<5$ years. These patients were probably considered to have a benign course based on negative cytology and/or the doctor's impression. As this study may have included cases in which evident malignant changes occurred during follow-up, the stenosis in most of the aforementioned patients could be benign. Therefore, the actual prevalence rate of malignancy might be lower.

MPD findings, including severity of stenosis, length of the stenotic site, and diameter of the upstream duct, did not differ among those with and without cancer in this study. In other words, it was extremely difficult to differentiate between neoplasm and non-neoplasm based on MPD findings. Therefore, histological confirmation is important. As EUS-guided fine needle aspiration cannot be applied because of absence of a mass, pancreatic juice cytology is the sole method for obtaining samples. Although a relatively favorable diagnostic yield was obtained in this study, further improvements are necessary. Iiboshi et al. have reported the usefulness of serial pancreatic-juice aspiration cytologic examination (SPACE) using a nasopancreatic catheter that is endoscopically inserted via the papilla. ${ }^{9}$ In their study, the sensitivity, specificity, and accuracy for detecting pancreatic cancer were $100 \%, 83 \%$, and $95 \%$, respectively, when pancreatic juice was intermittently sampled for an average of 5.3 times (range, 2-11 times) per person. Although SPACE appears to be the best way for sampling cells from the pancreatic duct at present, ${ }^{2,6-8,14,15}$ modifications for avoiding related issues, such as adverse events, discomfort in the nose, repetitive troublesome tasks, and requirement of experienced cytopathologists, might be needed before widespread application.

Appropriate performance and timing of ERP for evaluating FiCE, which has been confirmed to be related with pancreatic cancer with approximately $50 \%$ certainty, should be discussed. ERP evaluation is of no value in patients who are ineligible for surgical pancreatectomy. Patients with advanced age or with severe comorbidities that limit their prognoses do not require definite diagnosis using ERP because detection of early-stage cancer would not determine the prognoses of these patients. ERP would also not be required in patients in whom MPD findings have not changed over a long observation period, those with a history of severe pancreatitis that might have caused MPD irregularities, and those in whom previous cytological evaluation did not reveal malignancy. Contrastingly, ERP should be considered in young people, those without previous evaluations, those with high-risk factors for cancer (such as family history of pancreatic cancer), and those with- 
out strong risk factors for post-procedural pancreatitis (such as pancreatitis history). If ERP is not performed for evaluating FiCE, the patient should be carefully observed using dynamic CT, MRI, and EUS.

This retrospective study has limitations owing to the small sample size and single-center approach. Moreover, as mentioned before, this study also involved a selection bias; surgery was immediately performed on patients who were strongly suspected of having malignancies whereas other patients were observed and excluded if the follow-up period was insufficient. The actual prevalence of pancreatic neoplasms in patients with FiCE should be confirmed using prospective studies with large study populations.

In conclusion, the cause of a single, localized MPD stenosis was pancreatic cancer in $47 \%$ of patients with no other pancreatic disease or a detectable mass. Histological confirmation via pancreatic juice cytology is necessary to avoid unnecessary pancreatic resections.

\section{Conflicts of Interest}

The authors no financial conflicts of interest.

\section{Author Contributions}

Conceptualization: Yoshihide Kanno

Formal analysis: Shinsuke Koshita, Takahisa Ogawa, Hiroaki Kusunose, Kaori Masu, Toshitaka Sakai, Fumisato Kozakai, Yutaka Noda

Investigation: SK, TO, HK, KM, TS, Keisuke Yonamine, Yujiro Kawakami, Yuki Fujii, Kazuaki Miyamoto, Toji Murabayashi, FK, Jun Horaguchi, YN, Masaya Oikawa, Takaho Okada

Supervision: Kei Ito

Validation: KI

Writing-original draft: YK

Writing-review\&editing: KI

\section{REFERENCES}

1. Kamisawa T, Wood LD, Itoi T, Takaori K. Pancreatic cancer. Lancet 2016;388:73-85

2. Kanno A, Masamune A, Hanada K, et al. Multicenter study of early pancreatic cancer in Japan. Pancreatology 2018;18:61-67.

3. Cancer Information Service. Cancer statistics in Japan; Table download [Internet]. Tokyo: National Cancer Center; c2018 [updated 2018 Mar 1; cited 2018 Nov 8]. Available from: https:/ganjoho.jp/reg_stat/statistics/ $\mathrm{dl} /$ index.html.

4. Siegel RL, Miller KD, Jemal A. Cancer statistics, 2018. CA Cancer J Clin 2018;68:7-30.

5. Egawa S, Toma $\mathrm{H}$, Ohigashi $\mathrm{H}$, et al. Japan pancreatic cancer registry; 30th year anniversary: Japan Pancreas Society. Pancreas 2012;41:985992.

6. Hanada K, Okazaki A, Hirano N, et al. Diagnostic strategies for early pancreatic cancer. J Gastroenterol 2015;50:147-154.

7. Mouri T, Sasaki T, Serikawa M, et al. A comparison of 4-Fr with 5-Fr endoscopic nasopancreatic drainage catheters: a randomized, controlled trial. J Gastroenterol Hepatol 2016;31:1783-1789.

8. Hanada K, Amano H, Abe T. Early diagnosis of pancreatic cancer: current trends and concerns. Ann Gastroenterol Surg 2017;1:44-51.

9. Iiboshi T, Hanada K, Fukuda T, Yonehara S, Sasaki T, Chayama K. Value of cytodiagnosis using endoscopic nasopancreatic drainage for early diagnosis of pancreatic cancer: establishing a new method for the early detection of pancreatic carcinoma in situ. Pancreas 2012;41:523-529.

10. Miyata T, Takenaka M, Omoto S, et al. A case of pancreatic carcinoma in situ diagnosed by repeated pancreatic juice cytology. Oncology 2017;93 Suppl 1:98-101.

11. Cotton PB, Lehman G, Vennes J, et al. Endoscopic sphincterotomy complications and their management: an attempt at consensus. Gastrointest Endosc 1991;37:383-393.

12. Cotton PB, Eisen GM, Aabakken L, et al. A lexicon for endoscopic adverse events: report of an ASGE workshop. Gastrointest Endosc 2010;71:446-454.

13. Yokohata K, Shirahane K, Yonemasu H, et al. Focal ductal branch dilatation on magnetic resonance cholangiopancreatography: a hint for early diagnosis of pancreatic carcinoma. Scand J Gastroenterol 2000;35:12291232.

14. Satoh T, Kikuyama M, Kawaguchi S, Kanemoto H, Muro H, Hanada K. Acute pancreatitis-onset carcinoma in situ of the pancreas with focal fat replacement diagnosed using serial pancreatic-juice aspiration cytologic examination (SPACE). Clin J Gastroenterol 2017;10:541-545.

15. Hanada K, Okazaki A, Hirano N, et al. Effective screening for early diagnosis of pancreatic cancer. Best Pract Res Clin Gastroenterol 2015;29:929-939. 